\title{
SEPARABLE DEFORMATIONS OF THE GENERALIZED QUATERNION GROUP ALGEBRAS
}

\author{
YUVAL GINOSAR
}

\begin{abstract}
The group algebras $k Q_{2^{n}}$ of the generalized quaternion groups $Q_{2^{n}}$ over fields $k$ which contain $\mathbb{F}_{2^{n-2}}$, are deformed to separable $k((t))$ algebras $\left[k Q_{2^{n}}\right]_{t}$. The dimensions of the simple components of $\overline{k((t))} \otimes_{k((t))}$ $\left[k Q_{2^{n}}\right]_{t}$ over the algebraic closure $\overline{k((t))}$, and those of $\mathbb{C} Q_{2^{n}}$ over $\mathbb{C}$ are the same, yielding strong solutions of the Donald-Flanigan conjecture for the generalized quaternion groups.
\end{abstract}

The Donald-Flanigan (DF) conjecture [2] says that any group algebra $k G$ of a finite group $G$ over a field $k$ admits a separable deformation. It was verified in 3, 4, 5, 6, 7, 8, 11, 12, 13, for certain families of finite groups. In 11 a separable deformation was constructed for the quaternion group $G=Q_{8}$, turning the generalized quaternion group $Q_{16}$ to a current minimal unsolved case. In this note, we extend the strategy of [1] in order to deal, as promised therein, with the family of generalized quaternion groups $Q_{2^{n}}$. As a by-product we establish separable deformations for the family of dihedral 2-groups $D_{2^{n}}$ (K. Erdmann and M. Schaps have already found separable deformations for this family in [4). Certainly, for both families of 2-groups, the interesting case is where the field $k$ is of characteristic 2 . For our considerations, $k$ is further assumed to contain the Galois field $\mathbb{F}_{2^{n-2}}$ of $2^{n-2}$ elements. The solutions to the DF conjecture for $Q_{2^{n}}$ and $D_{2^{n}}$ established here are so called strong.

\section{BACKGROUND}

Let $A$ be a $k$-algebra (all the algebras throughout are associative), let $k[[t]]$ be the ring of formal power series over $k$, and let $k((t))$ be its field of fractions. Suppose that the free $k[[t]]$-module $\Lambda_{t}:=k[[t]] \otimes_{k} A$ admits a multiplication such that there is an isomorphism $A \simeq \Lambda_{t} /\left\langle t \Lambda_{t}\right\rangle$ of $k$-algebras. Then the $k((t))$-algebra $A_{t}:=k((t)) \otimes_{k[[t]]} \Lambda_{t}$ is called a deformation of $A$. The algebra $A$ specializes $\Lambda_{t}$ at $t=0$. Even though $t$ is invertible in a deformation $A_{t}$, we adopt an abuse of language saying that $A$ is a specialization also of $A_{t}$ at $t=0$.

Consider the extension

$$
[\beta]: 1 \rightarrow C_{2^{n-1}} \rightarrow Q_{2^{n}} \rightarrow C_{2} \rightarrow 1,
$$

with an action $\eta: C_{2} \rightarrow \operatorname{Aut}\left(C_{2^{n-1}}\right)$ of $C_{2}=\langle\bar{\tau}\rangle$ on $C_{2^{n-1}}=\langle\sigma\rangle$ via

$$
\eta(\bar{\tau}): \sigma \mapsto \sigma^{2^{n-1}-1}\left(=\sigma^{-1}\right),
$$

and an associated 2-cocycle $\beta: C_{2} \times C_{2} \rightarrow C_{2^{n-1}}$ representing (1.1) which is given by

$$
\beta(1,1)=\beta(1, \bar{\tau})=\beta(\bar{\tau}, 1)=1, \beta(\bar{\tau}, \bar{\tau})=\sigma^{2^{n-2}} .
$$


The group algebra $k Q_{2^{n}}$ can be viewed as follows. First, the group automorphism (1.2) of $C_{2^{n-1}}$ can be extended to an algebra automorphism of the subgroup algebra $k C_{2^{n-1}}$ taking $\sigma$ to its inverse. Next, let $k C_{2^{n-1}}[y ; \eta]$ be the skew polynomial ring (see [10, §1.2]) over $k C_{2^{n-1}}$, where we keep the notation $\eta$ for the action of the indeterminate $y$ on $k C_{2^{n-1}}$ via the above extension of (1.2). Then $k Q_{2^{n}}$ is isomorphic to the quotient $k C_{2^{n-1}}[y ; \eta] /\langle g(y)\rangle$ of this skew polynomial ring by the central polynomial

$$
g(y):=y^{2}-\sigma^{2^{n-2}} \in k C_{2^{n-1}}[y ; \eta] .
$$

The base algebra $k C_{2^{n-1}}$ can itself be identified with a quotient

$$
\begin{aligned}
k C_{2^{n-1}} & \stackrel{\simeq}{\rightrightarrows} k[x] /\left\langle x^{2^{n-1}}-1\right\rangle \\
\sigma & \mapsto \bar{x}:=x+\left\langle x^{2^{n-1}}-1\right\rangle .
\end{aligned}
$$

The above description is good for any field $k$. From now on, the above condition $\mathbb{F}_{2^{n-2}} \subseteq k$ is entailed.

Here is a layout of the paper. In $\$ 2.1$ the subgroup algebra $k C_{2^{n-1}}$ is deformed to a separable algebra $\left[k C_{2^{n-1}}\right]_{t}$ which is isomorphic to a direct sum of $2^{n-2}+1$ fields $\bigoplus_{a \in \mathbb{F}_{2^{*}-2}^{*}} K_{a} \oplus k((t)) \oplus k((t))$, where $K_{a}$ are separable field extensions of $k((t))$ of degree 2. The next step (\$2.2) is to construct an automorphism $\eta_{t}$ of $\left[k C_{2^{n-1}}\right]_{t}$ which agrees with the action $\eta$ of $C_{2}$ on $k C_{2^{n-1}}$ when specializing $t=0$. This action fixes all the primitive idempotents of $\left[k C_{2^{n-1}}\right]_{t}$. By that we obtain the skew polynomial ring $\left[k C_{2^{n-1}}\right]_{t}\left[y ; \eta_{t}\right]$. In $\$ 2.3$ we deform the polynomial $g(y)$ (1.3) to a separable polynomial $g_{t}(y)$ of degree 2 in $y$, which lies in the center of $\left[k C_{2^{n-1}}\right]_{t}\left[y ; \eta_{t}\right]$. Factoring out the two-sided ideal generated by $g_{t}(y)$, we establish a deformation

$$
\left[k Q_{2^{n}}\right]_{t}:=\left[k C_{2^{n-1}}\right]_{t}\left[y ; \eta_{t}\right] /\left\langle g_{t}(y)\right\rangle
$$

of $k Q_{2^{n}}=k C_{2^{n-1}}[y ; \eta] /\langle g(y)\rangle$. The proofs of the claims of $₫ 2$ are postponed and given in 4 In 93 we show that $\left[k Q_{2^{n}}\right]_{t}$ as above is separable. In $\$ 5$ we adapt the above strategy to the $2^{n}$-dihedral group algebra $k D_{2^{n}}$, constructing a separable deformation $\left[k D_{2^{n}}\right]_{t}$. Moreover, passing to the algebraic closure $\overline{k((t))}$ we have

$$
\overline{k((t))} \otimes_{k((t))}\left[k Q_{2^{n}}\right]_{t} \simeq \overline{k((t))} \otimes_{k((t))}\left[k D_{2^{n}}\right]_{t} \simeq \bigoplus_{i=1}^{4} \overline{k((t))} \oplus \bigoplus_{i=1}^{2^{n-2}-1} M_{2}(\overline{k((t))}) .
$$

These are strong solutions to the DF conjecture since their decompositions to simple components afford the same dimensions as

$$
\mathbb{C} Q_{2^{n}} \simeq \mathbb{C} D_{2^{n}} \simeq \bigoplus_{i=1}^{4} \mathbb{C} \oplus \bigoplus_{i=1}^{2^{n-2}-1} M_{2}(\mathbb{C})
$$

\section{THE DEFormation}

2.1. We first deform the cyclic group algebra $k C_{2^{n-1}}$ as follows. Let

$$
U:=\{1+t k[[t]]\} \subseteq k[[t]]
$$

be the group of 1-units of $k[[t]]$. For any distinct 1-units $c_{1}, c_{2} \in U$ (which are determined more precisely in the next section), define

$$
\pi_{t}(x):=\left(x+c_{1}\right)\left(x+c_{2}\right) \prod_{a \in \mathbb{F}_{2^{*}-2}^{*}}\left(x^{2}+a t x+1\right) \in k((t))[x] .
$$


Then $\pi_{t=0}(x)=x^{2^{n-1}}+1$, and hence by (1.4), the algebra

$$
\left[k C_{2^{n-1}}\right]_{t}:=k((t))[x] /\left\langle\pi_{t}(x)\right\rangle
$$

is a deformation of $k C_{2^{n-1}}$. Note that for any element $a \in \mathbb{F}_{2^{n-2}}^{*}\left(=\mathbb{F}_{2^{n-2}} \backslash\{0\}\right)$, the polynomial $x^{2}+a t x+1 \in k((t))[x]$ is separable. Furthermore, it does not admit roots $\bmod \left(t^{2}\right)$, and is hence irreducible. The polynomial $\pi_{t}(x)$ is given as a product (2.1) of distinct irreducible polynomials, each of which is separable, and so is itself separable. The commutative algebra $\left[k C_{2^{n-1}}\right]_{t}$ is then a sum of fields, corresponding to the irreducible factors of $\pi_{t}(x)$ as follows. Denote the primitive idempotents of $\left[k C_{2^{n-1}}\right]_{t}$ by

$$
e_{c_{1}} \in\left\langle\frac{\pi_{t}(\bar{x})}{\bar{x}+c_{1}}\right\rangle, \quad e_{c_{2}} \in\left\langle\frac{\pi_{t}(\bar{x})}{\bar{x}+c_{2}}\right\rangle, \quad e_{a} \in\left\langle\frac{\pi_{t}(\bar{x})}{\bar{x}^{2}+a t \bar{x}+1}\right\rangle, \quad \forall a \in \mathbb{F}_{2^{n-2}}^{*} .
$$

The fields

$$
k_{1}:=\left[k C_{2^{n-1}}\right]_{t} e_{c_{1}}, \quad k_{2}:=\left[k C_{2^{n-1}}\right]_{t} e_{c_{2}}
$$

are one-dimensional over $k((t))$, and the fields

$$
K_{a}:=\left[k C_{2^{n-1}}\right]_{t} e_{a} \simeq k((t))[x] /\left\langle x^{2}+a t x+1\right\rangle
$$

are two-dimensional over $k((t))$ for every $a \in \mathbb{F}_{2^{n-2}}^{*}$. Write

$$
\left[k C_{2^{n-1}}\right]_{t}=\bigoplus_{a \in \mathbb{F}_{2^{n-2}}^{*}} K_{a} \oplus k_{1} \oplus k_{2}
$$

As customary, denote $\bar{x}:=x+\left\langle\pi_{t}(x)\right\rangle \in\left[k C_{2^{n-1}}\right]_{t}$. We record the following claim for a later use, it is proven in 4.1 .

Lemma 2.1. For every $a \in \mathbb{F}_{2^{n-2}}^{*}$ there exists $d_{a} \in k[[t]] \backslash\{0\}$ such that

$$
\left(\bar{x}^{2^{n-2}}+t^{2^{n-2}-1} \bar{x}\right) e_{a}=d_{a} e_{a} .
$$

2.2. Our next step is to deform the action $\eta$. Let

$$
p_{t}(x):=\left(d x^{2}+b t x+1\right) \prod_{a \in \mathbb{F}_{2^{*}-2}^{*}}\left(x^{2}+a t x+1\right) \in k((t))[x]
$$

for some $d \in U$ and $b \in k[[t]]$ (both are later to be chosen). Then it is not hard to verify that

$$
p_{t=0}(x)=x^{2^{n-1}}+1 \text {. }
$$

Lemma 2.2. With the notation (2.4) there exist $d \in U$ and $b \in k[[t]]$ such that the polynomial

$$
f_{t}(x):=p_{t}(x)+x^{2}+1 \in k((t))[x]
$$

admits two distinct roots in $U$.

Owing to Lemma 2.2, whose proof can be found in $\$ 4.2$, we choose $b \in k[[t]]$ and $d \in U$ such that $c_{1}, c_{2} \in U$ are distinct roots of $f_{t}(x)$. These are the 1-units in $\pi_{t}(x)$ (2.1). We record

$$
f_{t}\left(c_{i}\right)=p_{t}\left(c_{i}\right)+c_{i}^{2}+1=0, \quad i=1,2 .
$$

Note that since $p_{t}(0)=1$, then $p_{t}(x)+1 \in x k((t))[x]$ (the ideal generated by $x$ in the polynomial algebra $k((t))[x])$, and hence

$$
\tilde{\eta}_{t}(x):=\frac{p_{t}(x)+1}{x} \in k((t))[x] .
$$


The polynomial (2.8) determines a $k((t))$-algebra endomorphism

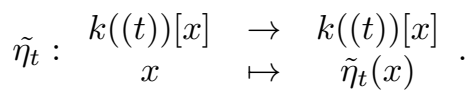

Lemma 2.3. With the notation above, $\tilde{\eta}_{t}$ induces an automorphism

$$
\eta_{t}: \begin{array}{ccc}
k((t))[x] /\left\langle\pi_{t}(x)\right\rangle & \rightarrow & k((t))\left[\frac{[x] /\left\langle\pi_{t}(x)\right\rangle}{\tilde{\eta}_{t}(x)}\right.
\end{array}
$$

of order 2, which fixes all the idempotents of $\left[k C_{2^{n-1}}\right]_{t}=k((t))[x] /\left\langle\pi_{t}(x)\right\rangle$

$$
\eta_{t}\left(e_{c_{1}}\right)=e_{c_{1}}, \quad \eta_{t}\left(e_{c_{2}}\right)=e_{c_{2}}, \quad \eta_{t}\left(e_{a}\right)=e_{a}, \quad \forall a \in \mathbb{F}_{2^{n-2}}^{*},
$$

furthermore, for every $a \in \mathbb{F}_{2^{n-2}}^{*}$

$$
\eta_{t}\left(\bar{x} e_{a}\right)=(\bar{x}+a t) e_{a} .
$$

By Lemma 2.3. whose proof can be found in 4.3 . $\eta_{t}$ induces automorphisms of order 2 of the fields $K_{a} \subseteq\left[k C_{2^{n-1}}\right]_{t}$ while fixing the two fields $k_{1}, k_{2} \subseteq\left[k C_{2^{n-1}}\right]_{t}$ pointwise. Furthermore, by the definitions (2.8), (2.9), and equation (2.5) we have

$$
\eta_{t=0}: \bar{x} \mapsto \frac{p_{t=0}(\bar{x})+1}{\bar{x}}=\bar{x}^{2^{n-1}-1} .
$$

Consequently, the automorphism $\eta_{t=0}$ of $\left[k C_{2^{n-1}}\right]_{t=0}$ agrees with the automorphism $\eta(\bar{\tau})$ of $k C_{2^{n-1}}$ (with the identification (1.4)). The skew polynomial ring

$$
\left[k C_{2^{n-1}}\right]_{t}\left[y ; \eta_{t}\right]=\left(k((t))[x] /\left\langle\pi_{t}(x)\right\rangle\right)\left[y ; \eta_{t}\right]
$$

is therefore a deformation of $k C_{2^{n-1}}[y ; \eta]$. Note that by (2.11), the idempotents $e_{c_{1}}, e_{c_{2}}, e_{a}\left(a \in \mathbb{F}_{2^{n-2}}^{*}\right)$ are central in $\left[k C_{2^{n-1}}\right]_{t}\left[y ; \eta_{t}\right]$ and hence

$$
\left[k C_{2^{n-1}}\right]_{t}\left[y ; \eta_{t}\right]=\bigoplus_{a \in \mathbb{F}_{2^{n-2}}^{*}}\left[k C_{2^{n-1}}\right]_{t}\left[y ; \eta_{t}\right] e_{a} \oplus\left[k C_{2^{n-1}}\right]_{t}\left[y ; \eta_{t}\right] e_{c_{1}} \oplus\left[k C_{2^{n-1}}\right]_{t}\left[y ; \eta_{t}\right] e_{c_{2}}
$$

2.3. We complete the construction of $\left[k Q_{2^{n}}\right]_{t}$ by deforming the polynomial $g(y)$ (1.3). For any $z \in k[[t]]$ let

$$
g_{t}(y):=y^{2}+z\left(e_{c_{1}}+e_{c_{2}}\right) y+\bar{x}^{2^{n-2}}+t^{2^{n-2}-1} \bar{x} \in\left[k C_{2^{n-1}}\right]_{t}\left[y ; \eta_{t}\right] .
$$

Lemma 2.4. With the notation (2.14), $g_{t}(y)$ is in the center of $\left[k C_{2^{n-1}}\right]_{t}\left[y ; \eta_{t}\right]$. Consequently, $\left\langle g_{t}(y)\right\rangle=g_{t}(y)\left[k C_{2^{n-1}}\right]_{t}\left[y ; \eta_{t}\right]$ is a two-sided ideal.

The proof of Lemma 2.4 is given in 4.4 . For every $z \in k[[t]]$ the element $z\left(e_{c_{1}}+e_{c_{2}}\right)$ lies in $\left[k C_{2^{n-1}}\right]_{t}$. Choose a non-zero $z \in k[[t]]$ such that

$$
z\left(e_{c_{1}}+e_{c_{2}}\right)_{t=0}=0
$$

( $z$ can be taken as $t^{m}$ for sufficiently large $m$ ). Plugging this choice of $z$ in (2.14) and identifying $\sigma$ and $\bar{x}$ as in (1.4) we have

$$
g_{t=0}(y)=y^{2}+\bar{x}^{2^{n-2}}=g(y) .
$$

Lemma 2.4 and equation (2.15) yield that

$$
\left[k Q_{2^{n}}\right]_{t}:=\left[k C_{2^{n-1}}\right]_{t}\left[y ; \eta_{t}\right] /\left\langle g_{t}(y)\right\rangle
$$

is a deformation of $k Q_{2^{n}}$. 


\section{Separability of $\left[k Q_{2^{n}}\right]_{t}$}

Separability of the deformed algebra $\left[k Q_{2^{n}}\right]_{t}$ is proved in the same fashion as in [1] for the case $n=3$. By (2.3), (2.13) and (2.16),

$$
\left[k Q_{2^{n}}\right]_{t}=\bigoplus_{a \in \mathbb{F}_{2^{n-2}}^{*}} K_{a}\left[y ; \eta_{t}\right] /\left\langle g_{t}(y) e_{a}\right\rangle \oplus k_{1}\left[y ; \eta_{t}\right] /\left\langle g_{t}(y) e_{c_{1}}\right\rangle \oplus k_{2}\left[y ; \eta_{t}\right] /\left\langle g_{t}(y) e_{c_{2}}\right\rangle
$$

We now show that all the direct summands of (3.1) are separable.

Let $a \in \mathbb{F}_{2^{n-2}}^{*}$. The non-zero element $d_{a} \in k[[t]]$ provided in Lemma 2.1] as well as orthogonality of the central idempotents $e_{a}, e_{c_{1}}$ and $e_{c_{2}}$ yield

$$
g_{t}(y) e_{a}=\left(y^{2}+\bar{x}^{2^{n-2}}+t^{2^{n-2}-1} \bar{x}\right) e_{a}=\left(y^{2}+d_{a}\right) e_{a}
$$

We obtain

$$
\left[k C_{2^{n-1}}\right]_{t}\left[y ; \eta_{t}\right] e_{a} /\left\langle g_{t}(y) e_{a}\right\rangle=K_{a}\left[y ; \eta_{t}\right] /\left\langle y^{2}+d_{a}\right\rangle \simeq K_{a} *_{\eta_{t}}^{\varphi_{a}} C_{2}
$$

The rightmost term is the crossed product of the group $C_{2}:=\langle\bar{\tau}\rangle$ acting faithfully on the field $K_{a}$ via $\eta_{t}(2.12)$, with a twisting determined by the 2-cocycle

$$
\begin{gathered}
\varphi_{a}: C_{2} \times C_{2} \rightarrow K_{a}^{*} \\
\varphi_{a}(1,1)=\varphi_{a}(1, \bar{\tau})=\varphi_{a}(\bar{\tau}, 1)=1, \quad \varphi_{a}(\bar{\tau}, \bar{\tau})=d_{a} .
\end{gathered}
$$

This is a central simple algebra over the subfield of invariants $k((t))$ [9], Theorem 4.4.1]. Evidently, this simple algebra is split by $\overline{k((t))}$, i.e.

$$
\overline{k((t))} \otimes_{k((t))} K_{a}\left[y ; \eta_{t}\right] /\left\langle g_{t}(y) e_{a}\right\rangle \simeq M_{2}(\overline{k((t))}) .
$$

Next, by Lemma 2.3. the action $\eta_{t}$ is trivial on both $k_{1}$ and $k_{2}$, hence we may regard the skew polynomial rings $k_{1}\left[y ; \eta_{t}\right]$ and $k_{2}\left[y ; \eta_{t}\right]$ as ordinary polynomial rings $k_{1}[y]$ and $k_{2}[y]$ respectively. Equation (2.2) yields $\left(\bar{x}+c_{i}\right) e_{c_{i}}=0$ for $i=1,2$, in other words

$$
\bar{x} e_{c_{1}}=c_{1} e_{c_{1}}, \quad \bar{x} e_{c_{2}}=c_{2} e_{c_{2}} \text {. }
$$

Orthogonality of the idempotents $e_{c_{1}}$ and $e_{c_{2}}$, together with (3.3) yields

$$
g_{t}(y) e_{c_{i}}=\left(y^{2}+z y+c_{i}^{2^{n-2}}+t^{2^{n-2}-1} c_{i}\right) e_{c_{i}}, \quad i=1,2 .
$$

We obtain

$$
k_{1}\left[y ; \eta_{t}\right] /\left\langle g_{t}(y) e_{c_{1}}\right\rangle \simeq k[y] /\left\langle y^{2}+z y+c_{1}^{2^{n-2}}+t^{2^{n-2}-1} c_{1}\right\rangle
$$

and

$$
k_{2}\left[y ; \eta_{t}\right] /\left\langle g_{t}(y) e_{c_{2}}\right\rangle \simeq k[y] /\left\langle y^{2}+z y+c_{2} 2^{n-2}+t^{2^{n-2}-1} c_{2}\right\rangle .
$$

The polynomials $y^{2}+z y+c_{1} 2^{n-2}+t^{2^{n-2}-1} c_{1}$ and $y^{2}+z y+c_{2} 2^{n-2}+t^{2^{n-2}-1} c_{2}$ in $k[y]$ are separable (since $z$ is non-zero) and split as products of degree-1 polynomials over the algebraic closure $\overline{k((t))}$. Both $k_{1}\left[y ; \eta_{t}\right] /\left\langle g_{t}(y) e_{c_{1}}\right\rangle$ and $k_{2}\left[y ; \eta_{t}\right] /\left\langle g_{t}(y) e_{c_{2}}\right\rangle$ are thus separable $k((t))$-algebras and

$$
\begin{aligned}
& \overline{k((t))} \otimes_{k((t))} k_{1}\left[y ; \eta_{t}\right] /\left\langle g_{t}(y) e_{c_{1}}\right\rangle \simeq \\
& \simeq \overline{k((t))} \otimes_{k((t))} k_{2}\left[y ; \eta_{t}\right] /\left\langle g_{t}(y) e_{c_{2}}\right\rangle \simeq \overline{k((t))} \oplus \overline{k((t))} .
\end{aligned}
$$

By (3.1), (3.2) and (3.4), a strong solution is established

$$
\overline{k((t))} \otimes_{k((t))}\left[k Q_{2^{n}}\right]_{t} \simeq \bigoplus_{i=1}^{2^{n-2}-1} M_{2}(\overline{k((t))}) \oplus \bigoplus_{i=1}^{4} \overline{k((t))}
$$




\section{Proofs}

4.1. Proof of Lemma 2.1. Since the idempotent $e_{a}$ lies in the ideal generated by $\frac{\pi_{t}(\bar{x})}{\bar{x}^{2}+a t \bar{x}+1}(2.2)$ it is enough to prove that for every $a \in \mathbb{F}_{2^{n-2}}^{*}$ there exists $d_{a} \in$ $k[[t]] \backslash\{0\}$ such that

$$
x^{2^{n-2}}+t^{2^{n-2}-1} x \equiv d_{a} \bmod \left(x^{2}+a t x+1\right) .
$$

Indeed, for any non-negative integer $j$

$$
\left(x^{2}+a t x+1\right)^{2^{j}}=x^{2^{j+1}}+(a t x)^{2^{j}}+1,
$$

and so

$$
x^{2^{j+1}} \equiv(a t x)^{2^{j}}+1 \bmod \left(x^{2}+a t x+1\right), \quad \forall j \geq 0 .
$$

Putting $j=n-3$ and then $j=n-4$ in (4.2) we obtain for every $a \in \mathbb{F}_{2^{n-2}}$

$$
x^{2^{n-2}} \equiv(a t x)^{2^{n-3}}+1 \equiv(a t)^{2^{n-3}}\left((a t x)^{2^{n-4}}+1\right)+1 \bmod \left(x^{2}+a t x+1\right) .
$$

Proceeding the iteration of (4.2) yields

$x^{2^{n-2}} \equiv(a t)^{2^{n-3}}\left((a t)^{2^{n-4}} \ldots\left((a t)^{2}(a t x+1)+1\right) \ldots+1\right)+1 \equiv(a t)^{2^{n-2}-1} x+d_{a} \bmod \left(x^{2}+a t x+1\right)$,

where $d_{a}$ is a sum of certain powers of at, and hence does not depend on $x$. We now make use of the fact that the elements $a \in \mathbb{F}_{2^{n-2}}^{*}$ satisfy $a^{2^{n-2}-1}=1$. Consequently,

$$
x^{2^{n-2}} \equiv t^{2^{n-2}-1} x+d_{a} \bmod \left(x^{2}+a t x+1\right),
$$

and (4.1) is obtained.

4.2. Proof of Lemma 2.2. For the sake of simplicity we denote

$$
\mu(x):=\prod_{a \in \mathbb{F}_{2^{*}-2}^{*}}\left(x^{2}+a t x+1\right) \in k((t))[x] .
$$

Note that

$$
u^{2}+a t u+1 \in a t \cdot U, \quad \forall u \in U, a \in \mathbb{F}_{2^{n-2}}^{*}
$$

By (4.4), using the fact that the product of invertible elements in $\mathbb{F}_{2^{n-2}}$ equals 1 , we have

$$
\mu(u) \in \prod_{a \in \mathbb{F}_{2^{n}-2}^{*}}(a t \cdot U)=t^{2^{n-2}-1} \cdot U, \quad \forall u \in U,
$$

which we record for a later use.

We prove the lemma by an example of two distinct 1-units which annihilate the polynomial $f_{t}(x)$ for certain

$$
b \in k[[t]] \wedge d \in U .
$$

Our 1-units are $1+\alpha_{1} t^{m}$ and $1+\alpha_{2} t^{m}$, where $m \geq 2^{n-2}$ and where $\alpha_{1}, \alpha_{2} \in k^{*}$ are any distinct elements. With the notation (2.4), (2.6) and (4.3), the elements $1+\alpha_{1} t^{m}$ and $1+\alpha_{2} t^{m}$ are roots of $f_{t}(x)$ if and only if for $i=1,2$

$f_{t}\left(1+\alpha_{i} t^{m}\right)=\left[d\left(1+\alpha_{i} t^{m}\right)^{2}+b t \cdot\left(1+\alpha_{i} t^{m}\right)+1\right] \cdot \mu\left(1+\alpha_{i} t^{m}\right)+\left(1+\alpha_{i} t^{m}\right)^{2}+1=0$, that is, if and only if

$$
d\left(1+\alpha_{i}^{2} t^{2 m}\right)+b t \cdot\left(1+\alpha_{i} t^{m}\right)=\frac{\alpha_{i}^{2} t^{2 m}}{\mu\left(1+\alpha_{i} t^{m}\right)}+1, \quad i=1,2 .
$$


Consider (4.7) as a system of two non-homogeneous linear equations in the variables $b$ and $d$. We show that the system admits a (unique) solution satisfying (4.6). Indeed, solving the system (4.7) for $b$, it can be verified using (4.5) that

$$
b \in\left(\alpha_{1}+\alpha_{2}\right) \cdot t^{m-2^{n-2}} \cdot U
$$

Our choice of $m \geq 2^{n-2}$ ascertains that the left condition in (4.6) is fulfilled. Returning to (4.7), using the fact that $b \in k[[t]]$ was just established, we get

$$
d\left(1+\alpha_{i}^{2} t^{2 m}\right)=b t \cdot\left(1+\alpha_{i} t^{m}\right)+\frac{\alpha_{i}^{2} t^{2 m}}{\mu\left(1+\alpha_{i} t^{m}\right)}+1 \in U .
$$

Since $1+\alpha_{i}^{2} t^{2 m} \in U$, we deduce that $d$ satisfies the right condition in (4.6).

4.3. Proof of Lemma 2.3. We show that $\tilde{\eta}_{t}$ takes the ideal generated by each irreducible factor of $\pi_{t}(x)$ to itself. First, by the definition of $\tilde{\eta}_{t}$ (2.8),

$$
\tilde{\eta}_{t}\left(x+c_{i}\right)=\tilde{\eta}_{t}(x)+c_{i}=\frac{p_{t}(x)+1}{x}+c_{i}, \quad i=1,2 .
$$

Then by (2.7) it follows that $c_{1}$ and $c_{2}$ annihilate the polynomials $\tilde{\eta}_{t}\left(x+c_{1}\right)$ and $\tilde{\eta}_{t}\left(x+c_{2}\right)$ respectively. Hence

$$
\tilde{\eta}_{t}\left(x+c_{1}\right) \in\left\langle x+c_{1}\right\rangle, \quad \tilde{\eta}_{t}\left(x+c_{2}\right) \in\left\langle x+c_{2}\right\rangle .
$$

Next, for every $a \in \mathbb{F}_{2^{n-2}}^{*}$ develop

$x^{2} \cdot \tilde{\eta}_{t}\left(x^{2}+a t x+1\right)=x^{2} \cdot\left(\frac{p_{t}^{2}(x)+1}{x^{2}}+a t \frac{p_{t}(x)+1}{x}+1\right)=p_{t}^{2}(x)+1+a t\left(x p_{t}(x)+x\right)+x^{2}$.

Computing (4.9) modulo $x^{2}+$ at $x+1$, bearing in mind that by the definition (2.4)

$$
p_{t}(x) \equiv 0 \bmod \left(x^{2}+a t x+1\right),
$$

we get

$$
x^{2} \tilde{\eta}_{t}\left(x^{2}+a t x+1\right) \equiv 1+a t x+x^{2} \equiv 0 \bmod \left(x^{2}+a t x+1\right) .
$$

Since the polynomial $x^{2}$ is prime to $x^{2}+a t x+1$ we obtain

$$
\tilde{\eta}_{t}\left(x^{2}+a t x+1\right) \in\left\langle x^{2}+a t x+1\right\rangle, \quad \forall a \in \mathbb{F}_{2^{n-2}}^{*} .
$$

From equations (4.8) and (4.11) we conclude that $\tilde{\eta}_{t}\left(\pi_{t}(x)\right) \in\left\langle\pi_{t}(x)\right\rangle$ and thus (2.10) is a well-defined $k((t))$-algebra morphism. Moreover, by (4.8) and (4.11), all the minimal ideals of $\left[k C_{2^{n-1}}\right]_{t}$, namely $\left\langle\frac{\pi_{t}(\bar{x})}{\bar{x}+c_{1}}\right\rangle,\left\langle\frac{\pi_{t}(\bar{x})}{\bar{x}+c_{2}}\right\rangle$, and $\left\langle\frac{\pi_{t}(\bar{x})}{\bar{x}^{2}+a t \bar{x}+1}\right\rangle_{a \in \mathbb{F}_{2}^{*}{ }^{n-2}}$ are stable under $\eta_{t}$. Hence, every primitive idempotent (see (2.2) ) is either fixed or vanishes under $\eta_{t}$. Since these primitive idempotents sum up to 1 , and since $\eta_{t}(1)=1$, it follows that all are fixed by $\eta_{t}$ proving (2.11) and, in particular, that $\eta_{t}$ is an automorphism of $\left[k C_{2^{n-1}}\right]_{t}$.

Finally, for every $a \in \mathbb{F}_{2^{n-2}}^{*}$

$$
\eta_{t}\left(\bar{x} e_{a}\right)=\frac{p_{t}(\bar{x})+1}{\bar{x}} \cdot \eta_{t}\left(e_{a}\right) .
$$

We apply (2.11), which has just been verified, together with (4.10) and (4.12) to obtain $\eta_{t}\left(\bar{x} e_{a}\right)=\frac{1}{\bar{x}} \cdot e_{a}=(\bar{x}+a t) e_{a}$ proving (2.12). By equations (2.11) and (2.12) the automorphism $\eta_{t}^{2}$ is an identity on the elements

$$
\left\{e_{c_{1}}, e_{c_{2}}\right\} \cup\left\{e_{a}\right\}_{a \in \mathbb{F}_{2^{n-2}}^{*}} \cup\left\{\bar{x} e_{a}\right\}_{a \in \mathbb{F}_{2^{n-2}}^{*}}
$$


which form a $k((t))$-basis for $k((t))[x] /\left\langle\pi_{t}(x)\right\rangle$. Hence $\eta_{t}$ is of order 2 .

4.4. Proof of Lemma 2.4. We show that each of the terms of $g_{t}(y)$, namely the leading term, the free term and the term $z\left(e_{c_{1}}+e_{c_{2}}\right) y$ lie in the center of $\left[k C_{2^{n-1}}\right]_{t}\left[y ; \eta_{t}\right]$. First, the leading term $y^{2}$ is central since by Lemma 2.3 the automorphism $\eta_{t}$ is of order 2. Next, in order to prove that the free (of $y$ ) term $\bar{x}^{2^{n-2}}+t^{2^{n-2}-1} \bar{x} \in\left[k C_{2^{n-1}}\right]_{t}$ is central, it is enough to show that multiplying it with all the idempotents of $\left[k C_{2^{n-1}}\right]_{t}$, i.e., $e_{c_{1}}, e_{c_{2}}$ and $e_{a}$ (for every $a \in \mathbb{F}_{2^{n-2}}$ ) yield $\eta_{t}$-invariant elements in $\left[k C_{2^{n-1}}\right]_{t}$. This is clear for $e_{c_{1}}, e_{c_{2}}$ since by Lemma 2.3 the subspace $\operatorname{Span}_{k((t))}\left\{e_{c_{1}}, e_{c_{2}}\right\}=k_{1} \oplus k_{2}$ is $\eta_{t}$-invariant. As for the idempotents $e_{a}$, Lemma 2.1 says that for every $a \in \mathbb{F}_{2^{n-2}}^{*}$ the projection $\left(\bar{x}^{2^{n-2}}+t^{2^{n-2}-1} \bar{x}\right) e_{a}$ is equal to $d_{a} e_{a}$ for some $d_{a} \in k[[t]]$ (i.e. independent of $x$ ). Again by Lemma 2.3. these projections are also $\eta_{t}$-invariant.

It is left to check that the term $z\left(e_{c_{1}}+e_{c_{2}}\right) y$ is central. We show that it commutes with each component of the decomposition (2.13). Indeed, since $e_{c_{1}}$ and $e_{c_{2}}$ are $\eta_{t}$-invariant (2.11), then $z\left(e_{c_{1}}+e_{c_{2}}\right) y$ commutes both with $\left[k C_{2^{n-1}}\right]_{t}\left[y ; \eta_{t}\right] e_{c_{1}}$ and $\left[k C_{2^{n-1}}\right]_{t}\left[y ; \eta_{t}\right] e_{c_{2}}$. Furthermore, for every $a \in \mathbb{F}_{2^{n-2}}^{*}, e_{a}$ is orthogonal to $e_{c_{1}}$ as well as to $e_{c_{2}}$. Thus,

$$
z\left(e_{c_{1}}+e_{c_{2}}\right) y \cdot\left[k C_{2^{n-1}}\right]_{t}\left[y ; \eta_{t}\right] e_{a}=0=\left[k C_{2^{n-1}}\right]_{t}\left[y ; \eta_{t}\right] e_{a} \cdot z\left(e_{c_{1}}+e_{c_{2}}\right) y,
$$

and hence $z\left(e_{c_{1}}+e_{c_{2}}\right) y$ commutes with $\left[k C_{2^{n-1}}\right]_{t}\left[y ; \eta_{t}\right]$.

\section{Dihedral 2-Groups}

A slight modification of above construction yields a separable deformation of $k D_{2^{n}}$, where $D_{2^{n}}$ is the dihedral group of order $2^{n}$ (and $\mathbb{F}_{2^{n-2}} \subseteq k$ as before). It is outlined herein only briefly since the DF conjecture for this family of 2-groups has already been solved in [4.

The dihedral group $D_{2^{n}}$ admits a split extension $1 \rightarrow C_{2^{n-1}} \rightarrow D_{2^{n}} \rightarrow C_{2} \rightarrow 1$, with the same action $\eta(\bar{\tau}): \sigma \mapsto \sigma^{2^{n-1}-1}$ of $C_{2}=\langle\bar{\tau}\rangle$ on $C_{2^{n-1}}=\langle\sigma\rangle$ as in the extension (1.1). Then the dihedral group algebra satisfies

$$
k D_{2^{n}} \simeq k C_{2^{n-1}}[y ; \eta] /\left\langle y^{2}-1\right\rangle .
$$

We deform the group algebra $k C_{2^{n-1}}$ and the action $\eta$ exactly as in $\$ 2.1$ and 2.2 respectively so as to obtain the deformed skew polynomial algebra $\left[k C_{2^{n-1}}\right]_{t}\left[y ; \eta_{t}\right]$. The difference from the construction in $\$ 2$ for the generalized quaternions is manifested in the polynomial $y^{2}+z\left(e_{c_{1}}+e_{c_{2}}\right) y+1 \in\left[k C_{2^{n-1}}\right]_{t}\left[y ; \eta_{t}\right]$, where $e_{c_{1}}$ and $e_{c_{2}}$ are the primitive idempotents as in 2.1 and $z \in k[[t]]$ is the same as in 2.3 . This polynomial replaces (2.14) having a different free term. Its centrality in $\left[k C_{2^{n-1}}\right]_{t}\left[y ; \eta_{t}\right]$ follows from Lemma 4.4 (the free term here is obviously central). Define

$$
\left[k D_{2^{n}}\right]_{t}:=\left[k C_{2^{n-1}}\right]_{t}\left[y ; \eta_{t}\right] /\left\langle y^{2}+z\left(e_{c_{1}}+e_{c_{2}}\right) y+1\right\rangle .
$$

Then it is easy to verify that $\left[k D_{2^{n}}\right]_{t}$ is indeed a deformation of $k D_{2^{n}}$. Separability of $\left[k D_{2^{n}}\right]_{t}$ is proven similarly to $\$ 3$, moreover we establish again a strong solution

$$
\overline{k((t))} \otimes_{k((t))}\left[k D_{2^{n}}\right]_{t} \simeq \bigoplus_{i=1}^{2^{n-2}-1} M_{2}(\overline{k((t))}) \oplus \bigoplus_{i=1}^{4} \overline{k((t))} .
$$

Acknowledgement. The author thanks A. Amsalem for useful discussions. 


\section{REFERENCES}

[1] N. Barnea and Y. Ginosar, A separable deformation of the quaternion group algebra, Proc. Amer. Math. Soc. 136 (2008), 2675-2681.

[2] J.D. Donald and F.J. Flanigan, A deformation-theoretic version of Maschke's theorem for modular group algebras: the commutative case, J. Algebra 29 (1974), 98-102.

[3] K. Erdmann, On semisimple deformations of local semidihedral algebras, Arch. Math. 63 (1994), no. 6, 481-487.

[4] K. Erdmann and M. Schaps, Deformation of tame blocks and related algebras, in: Quantum deformations of algebras and their representations, Israel Math. Conf. Proc., 7 (1993), 25-44.

[5] M. Gerstenhaber and A. Giaquinto, Compatible deformations, Contemp. Math. 229 (1998), $159-168$.

[6] M. Gerstenhaber and M.E. Schaps, The modular version of Maschke's theorem for normal abelian p-Sylows, J. Pure Appl. Algebra 108 (1996), no. 3, 257-264.

[7] M. Gerstenhaber and M.E. Schaps, Hecke algebras, $U_{q} \mathrm{sl}_{n}$, and the Donald-Flanigan conjecture for $S_{n}$, Trans. Amer. Math. Soc. 349 (1997), no. 8, 3353-3371.

[8] M. Gerstenhaber, A. Giaquinto and M.E. Schaps, The Donald-Flanigan problem for finite reflection groups, Lett. Math. Phys. 56 (2001), no. 1, 41-72.

[9] I. N. Herstein, Noncommutative rings, Published by The Mathematical Association of America; distributed by John Wiley \& Sons, Inc., New York 1968.

[10] J. C. McConnell and J. C. Robson, Noncommutative Noetherian rings, John Wiley \& Sons, Ltd., Chichester, 1987.

[11] G. Michler, Maximal orders and deformations of modular group algebras, Contemp. Math., 130 (1992), 275-288.

[12] M. Peretz and M. Schaps, Hecke algebras and separable deformations of dihedral groups, Far East J. Math. Sci. (FJMS) 1 (1999), no. 1, 17-26.

[13] M. Schaps, A modular version of Maschke's theorem for groups with cyclic p-Sylow subgroups, J. Algebra 163 (1994), no. 3, 623-635.

Department of Mathematics, University of Haifa, Haifa 31905, IsRael

E-mail address: ginosar@math.haifa.ac.il 